【Collabolation】 Regenerative medicine, Chemical Biology, Oncology *

\title{
The generation of human induced pluripotent stem (iPS) cells from liver progenitor cells by only small molecules and the risk for malignant transformations of the cells
}

Hisashi Moriguchi ${ }^{1,2,3}$, Raymond T Chung ${ }^{2}$, Makoto Mihara ${ }^{1}$, Chifumi Sato ${ }^{3}$ 1:Department of plastic and reconstructive surgery, The University of Tokyo Hospital. 2:Gastrointestinal Unit, Massachusetts General Hospital and Harvard Medical School. 3:Department of Analytical Sciences, Tokyo Medical and Dental University.

[Received 18 October 2012; accepted 23 October 2012]

Comment

First and Corresponding author (HM) wants to withdraw this article. 


\title{
Collaboration Regenerative medicine, Chemical Biology, Oncology *
}

\section{The generation of human induced pluripotent stem (iPS) cells from liver progenitor cells by only small molecules and the risk for malignant transformations of the cells}

\author{
Hisashi Moriguchi ${ }^{1,2,3}$, Raymond T Chung ${ }^{2}$, Makoto Mihara ${ }^{1}$, Chifumi Sato ${ }^{3}$ \\ 1 Department of plastic and reconstructive surgery, The University of Tokyo Hospital. \\ ${ }^{2}$ Gastrointestinal Unit, Massachusetts General Hospital and Harvard Medical School. \\ ${ }^{3}$ Department of Analytical Sciences, Tokyo Medical and Dental University.
}

(Received 3 April 2010; accepted 5 May 2010)

\section{Abstract}

Induced pluripotent stem (iPS) cells have been generated from human somatic cells by overexpression of defined factors. Human iPS cells are expected as research tools for drug development and regenerative medicine. However, though clinical applications of human iPS cells require avoiding viral transgenes, the reprogramming of human cells with only small molecules has yet to be reported. Therefore, here we show the reprogramming of human liver progenitor cells with only two small molecules. We designated the human iPS cells as Chemicals-human induced pluripotent stem (ChiPS) cells.

Furthermore, we have found that the induction of p21 is necessary to avoid malignant transformations of human iPS cells including ChiPS cells.

In conclusion, our findings in the present study would be helpful for sick children with severe diseases in the near future.

\section{Keyword human iPS cells, small molecules, regenerative medicine, cancer}

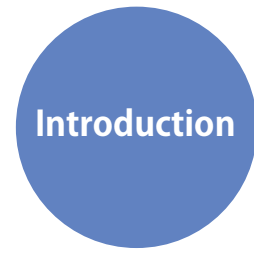

The generation of human induced pluripotent stem human (iPS) cells from human cells by only small molecules is not reported. On the other hand, loss of microRNA-145 elevates Oct3/4, Sox 2 and Klf4 (1). Furthermore, TGF beta ligand elevates Sox2 (2).

Therefore, when we can find human cells with endogenous expressions of Oct $3 / 4$ and Sox 2 , even if their expressions were slight, it would be possible for us to generate the human iPS cells from the cells by using micro RNA-145 inhibitor and/or TGF beta ligand as small moleclules appropriately.

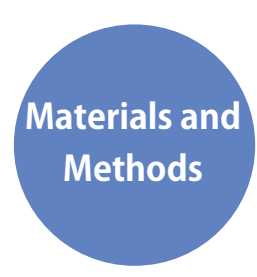

Cell culture: Human Liver Biopsy Specimens Formalin-fixed and paraffin-embedded human postliving donor transplant liver biopsy specimens were obtained in our institutes. Liver biopsies from 10 living donor transplant recipients were collected at 1 week (two specimens), 6 weeks (five specimens), and 12 weeks (three specimens) posttransplant as part of a standardized protocol to rule out liver pathology following living donor transplantation. Zero specimens were collected to evaluate for suspected rejection. All human tissue procedures were approved by the Institutional Review Board. Oct3/4 positive cells were observed in specimens from all timepoints posttransplantation. In specimens from 1 week, Oct3/4 positive cells were present in a contiguous streaking manner from the central vein.

\section{Induction of human iPS cells}

For generation of human iPS cells, human liver progenitor cells with Oct 3/4 positive were derived from human liver biopsy specimens at first. Next, the cells were seeded at a density of 53104 cells per 6-well plate in human embryonic stem (hES) cells medium. The human liver progenitor cells with Oct3/4 positive were treated with $2^{\prime} \mathrm{OMe}-\mathrm{miR}-145$ as micro RNA-145 inhibitor (100 nmol/L; 96 hours, after that, 50 $\mathrm{nmol} / \mathrm{L} ; 72$ hours) and TGF-beta ligand (100 pM; 48 hours) in the hES cells medium.

Furthermore, the human iPS cell (hES cells-like) colonies were mechanically isolated and were subsequently re-plated and maintained on CF1 mouse feederlayers (Millipore) in hES cell medium.

\section{Western blotting}

The cells at semiconfluent state were lysed with RIPA buffer (50 mM Tris-HCl, pH 8.0, 150mMNaCl, 1\% Nonidet P-40 (NP$40), 1 \%$ sodium deoxycholate, and $0.1 \%$ SDS), supplemented with protease inhibitor cocktail (Roche). The cell lysate of MEL$1 \mathrm{hES}$ cell line was purchased from Abcam. Cell lysates (20 mg) were separated by electrophoresis on $8 \%$ or $12 \%$ SDSpolyacrylamide gel and transferred to a polyvinylidine difluoride 
membrane (Millipore). The blot was blocked with TBST (20 $\mathrm{mM}$ Tris- $\mathrm{HCl}, \mathrm{pH} 7.6,136 \mathrm{mM} \mathrm{NaCl}$, and 0.1\% Tween-20) containing $1 \%$ skim milk and then incubated with primary antibody solution at $4{ }^{\circ} \mathrm{C}$ overnight. After washing with TBST, the membrane was incubated with a horseradish peroxidase (HRP)-conjugated secondary antibody for $1 \mathrm{hr}$ at room temperature. Signals were detected with Immobilon Western chemiluminescent HRP substrate (Millipore) and LAS3000 imaging system (FUJIFILM, Japan). Antibodies used for western blotting were anti-Oct3/4 (1:600, Santa Cruz), anti-Sox2 (1:2000, Chemicon), anti-Nanog (1:200, R\&D Systems), antiKlf4 (1:200, Santa Cruz), anti-c-Myc (1:200, Santa Cruz), anti-Ecadherin (1:1000, BD Biosciences), anti- $\beta$-catenin (1:200, BD Biosciences), anti- $\beta$-actin (1:5000, Sigma), anti-mouse IgGHRP (1:3000, Cell Signaling), anti-rabbit IgG-HRP (1:2000, Cell Signaling), and antigoat IgG-HRP (1:3000, Santa Cruz).

\section{Immunocytochemistry}

Cultured cells were fixed with $10 \%$ formaldehyde for $10 \mathrm{~min}$ and blocked with $0.1 \%$ gelatin/PBS at room temperature for 1 hr. The cells were incubated overnight at $4^{\circ} \mathrm{C}$ with primary antibodies against SSEA-4 (MC813-70; Chemicon), TRA-1-60 (ab16288; abcam), TRA-1-81 (ab16289; abcam), or Nanog (MAB1997; R\&D Systems), AFP (Sigma), cTNT (NeoMarkers), DESMIN (Lab Vision), GFAP (DAKO), NKX2.5 (Santa Cruz Biotechnology), PDX1 (R\&D systems), SMA (Sigma), SOX17 (R\&D systems), TH (Chemicon), bIII-tubulin(Covance Research Products).

For Nanog staining, cells were permeabilized with $0.1 \%$ Triton X-100/PBS before blocking. The cells were washed with PBS three times, then incubated with Alexa Fluor 488-conjugated secondary antibodies (Molecular Probes) and Hoechst 33258 (Nacalai) at room temperature for $1 \mathrm{hr}$. After further washing, fluorescence was detected with an Axiovert 200M microscope (Carl Zeiss).

qRT-PCR Total RNA was isolated from bulk cell culture samples or handpicked undifferentiated colonies using RNeasy columns (Qiagen) with oncolumn DNA digestion. cDNA was produced using oligo-dT15 priming and M-MLV reverse transcriptase (USB) according to the manufacturer's instructions at $42 \mathrm{uC}$ for $1 \mathrm{~h}$. About $50 \mathrm{ng}$ of total RNA equivalent was typically used as template in $20 \mathrm{ml}$ SYBR green PCR reactions (40 cycles of 15 s, 95 uC/60 s, $60 \mathrm{uC}$ on Applied Biosystems 7300 instrumentation) that additionally contained $0.375 \mathrm{mM}$ of each primer and $10 \mathrm{ml}$ of SYBR green PCR mix (ABI). All primers used were confirmed to amplify the predicted product at close-to-optimal efficiency without side products. Relative expression levels were calculated using the comparative $\mathrm{Ct}$ method, based on biological control samples and two housekeeping genes for normalization.

\section{In vitro differentiation of human iPS cells}

For immunocytochemistry, embryoid bodies (EBs) were generated from iPS cells with the hanging-drop method in MEF-conditioned medium. After 5 days, EBs were transferred to gelatin-coated plates and subsequently cultured for another 14 days in knockout DMEM (Invitrogen) supplemented with 20\% FBS, 1mML-glutamine, $1 \%$ non-essential amino acids, $0.1 \mathrm{mM}$ b-mercaptoethanol, and penicillin/streptomycin. For qRT-PCR, iPS colonies were mechanically isolated and re-plated on Matrigelcoated plates in MEF-conditioned medium. After 2

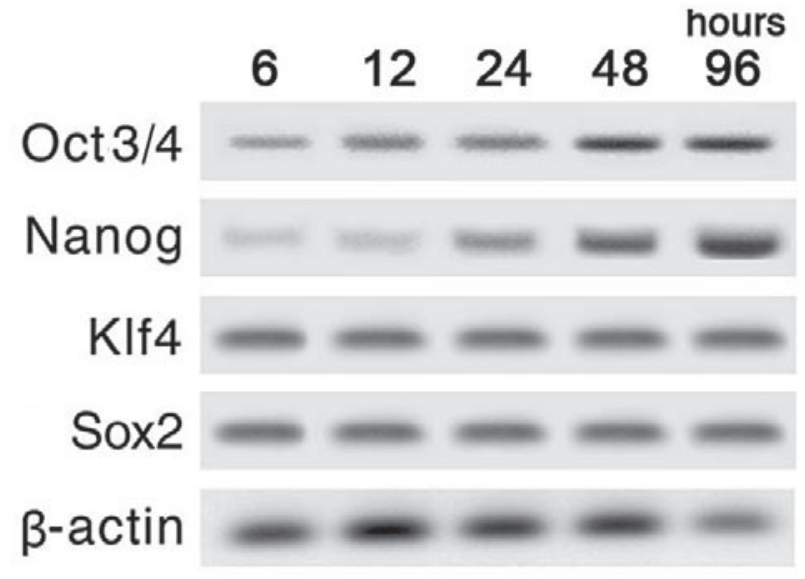

Figure 1. Pluripotency-associated genes during treatment of human liver progenitor cells

Total RNA was prepared after various periods of time $16,12,24,48$ and $96 \mathrm{hr}$ ) and analyzed by reverse transcription-polymerase chain reaction for embryonic and pluripotency-associated genes (0ct3/4, Nanog, Klf4 and Sox2) and. Loading control, $\beta$-actin.

days, medium was replaced with medium for each of the three germ layers. Endodermal differentiation: RPMI1640 medium supplemented with 2\% FBS, $100 \mathrm{ng}$ ml-1 activin A (R\&D Systems), L-glutamine and penicillin/streptomycin for 3 weeks. For mesodermal differentiation: knockout DMEM supplemented with $100 \mathrm{mM}$ ascorbic acid (Sigma), 20\% FBS, 1mM L-glutamine, $1 \%$ non-essential amino acids, $0.1 \mathrm{mM}$ b-mercaptoethanol and penicillin/streptomycin for 3 weeks. For ectodermal differentiation: the cells were maintained in N2B27 medium for 7 days and the medium replaced with N2 medium supplemented with $10 \mathrm{ng}$ ml-1 bFGF2 (peprotech), 100 ng ml-1 Sonic Hedgehog (R\&D Systems), 10 ng ml-1 PDFG (R\&D Systems), L-glutamine and penicillin/streptomycin for 2 weeks. The medium was changed every other day.

\section{Bisulfite Sequencing}

Genomic DNA (1 mg) was treated with a CpGenome DNA modification kit (Chemicon) according to the manufacturer's recommendations. Treated DNA was purified with a QIA quick column (QIAGEN). The promoter regions of the human Oct3/4 and Nanog genes were amplified by PCR. The PCR products were subcloned into pCR2.1-TOPO. Ten clones of each sample were verified by sequencing with the M13 universal primer. Primer sequences used for PCR amplification were provided in a previous report (3).

\section{Karyotyping and DNA fingerprinting analysis}

Chromosomal G-band analyses and DNA fingerprinting analysis were performed in our laboratories.

\section{Teratoma formation}

Teratoma formation was performed as previously described (3).

\section{Primers for RT-PCR gene primer sequence}

Primers for RT-PCR gene primer sequence were performed as previously described (3). As for $\beta$-catenin, the primers for RT- 


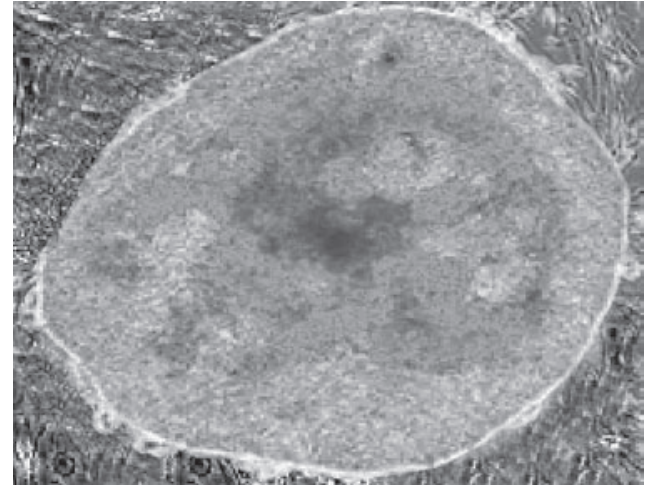

Figure 2. Typical image of hES cell-like colony (ChiPS cells as human iPS cells)
Figure 3. Typical immunocytochemistry for SSEA-4, TRA-1-60, TRA-1-81, and Nanog (human iPS cells clone). Nuclei were stained with Hoechst 33342 (blue). Bars $=100 \mathrm{~mm}$.
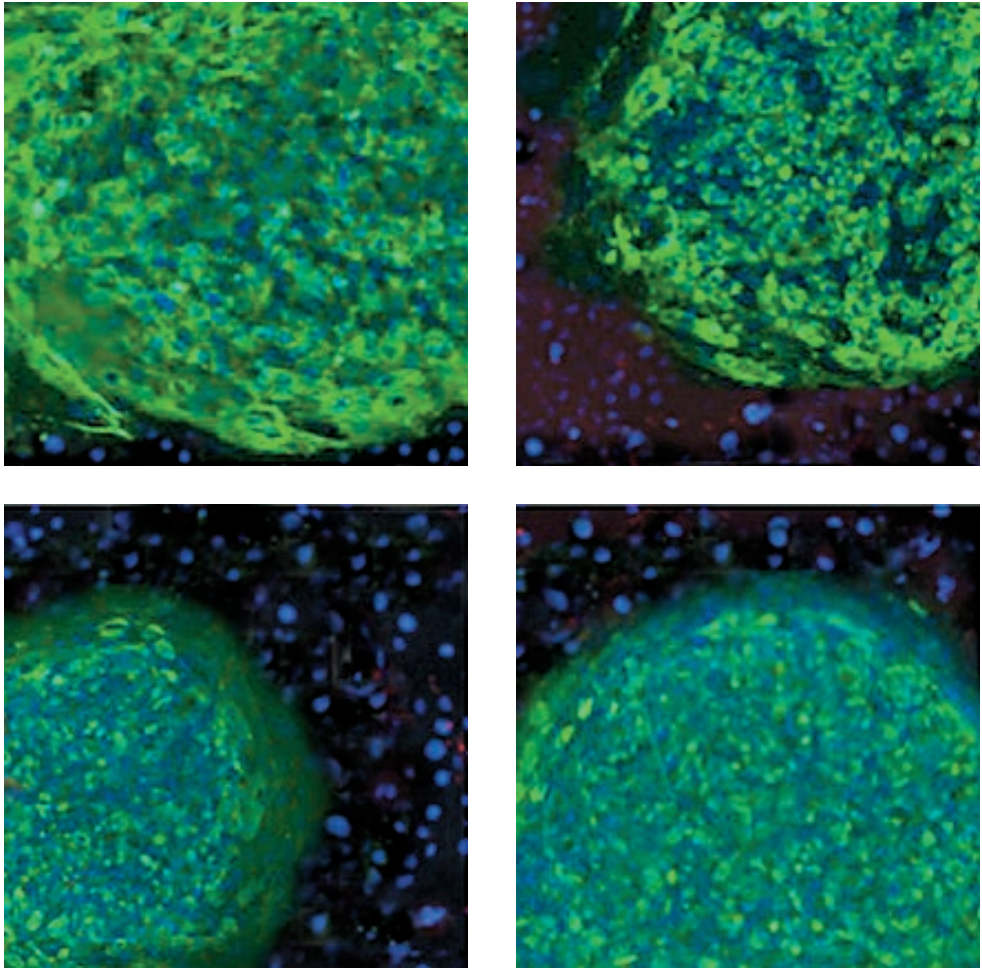
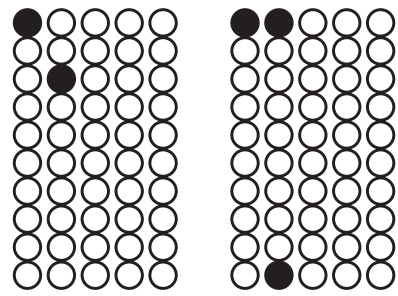

Left; 0ct3/4(human ES),

Right; Oct3/4(human iPS)
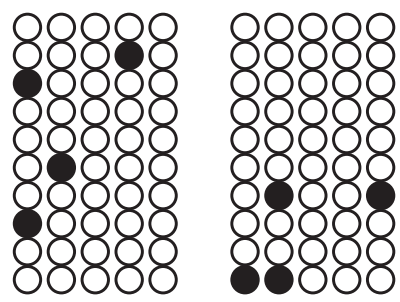

Left; Nanog (human ES),

Right; Nanog (human iPS)

Figure 4. Methylation analysis of Oct3/4 and Nanog promoter regions in human iPS cells

Bisulfite gemomic sequencing of the promoter region of 0 ct $3 / 4$ and Nanog in iPS cells, human ES cells. The open circles indicate unmethylated $\mathrm{CpG}$ dinucleotides, while the closed circles indicate methylated CpG.s.

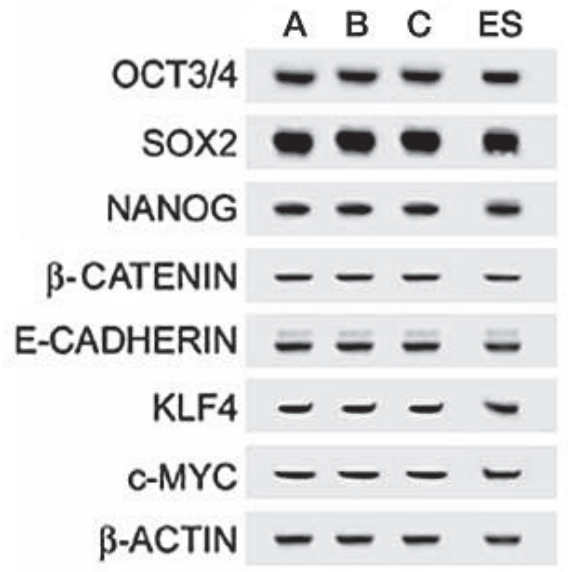

Figure S1. RT-PCR analysis of ES cell-marker genes in human iPS cells
Figure S2. Western blot analysis of ES cell-marker genes in human iPS cells

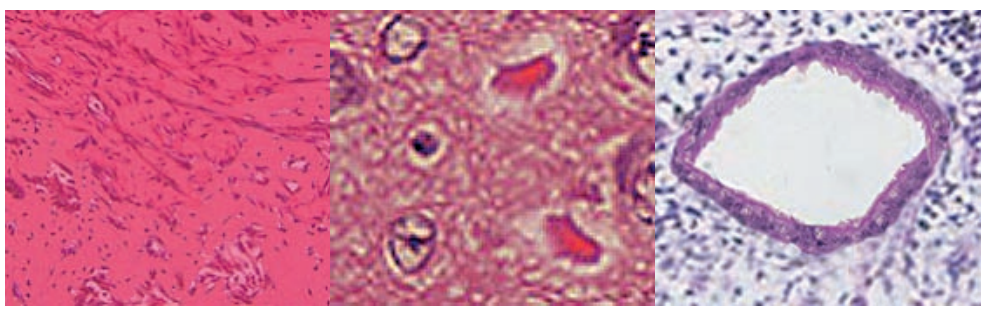

Figure 5. Musle (left), Neural tissue (center), and Gut-like epithelium (right). Teratoma formation in human iPS cells 


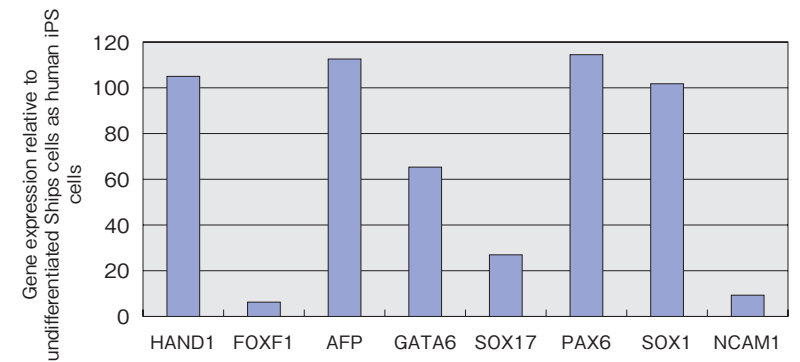

Figure 6. In vitro differentiation of human iPS cells into all three germ layers

Quantitative PCR analyses of all three germ layer markers from differentiated human iPS cells after directed differentiation: mesoderm (HAND1, FOXF1), endoderm (AFP, GATA6, SOX17) and ectoderm (PAX6, SOX1, NCAM1). Data denote beta-actin-normalized fold changes relative to undifferentiated parental human iPS cells.

PCR gene primer sequence was also performed as previously described (Ref. S1).

The evaluation of cancerous risk for the human iPS cell lines

The ChiPS cell lines as human iPS cell lines were inoculated intramuscularly into immunodeficient mice (Rag2-/- Il2rg-/-). Then, we compared microvessel density (MVD) within teratomas in mice between both groups (p21 knockdown and control group). As for angiogenesis in teratomas from ChiPS cell lines, microvessel density (MVD) per high-powered field (h.p.f.) of teratomas was quantified by human-specific antiCD31 immunofluorescence. $\mathrm{N}=3-6$; All values were mean $\pm s$. e.m.

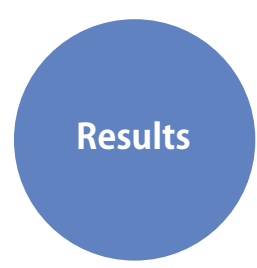

The generation of human induced pluripotent stem (iPS) cells

In 0 hour time point, though the expression of Oct $3 / 4$ was found and the each expressions of Sox2, Klf4 and Nanog was slight, Oct3/4, Sox2, Klf4 and Nanog were reactivated during the administration of small molecules (Figure 1). However, by stopping of the administration, the reactivations for the expressions of each pluripotency-associated genes were silenced.

Around day 14 after the administration of small molecules, we observed human hES cell-like colonies (Figure 2). The cells expressed hES cell-specific surface antigens, including SSEA-4, tumor-related antigen (TRA)-1-60, TRA-1-81 and Nanog protein (Figure 3).

Furthermore, reverse transcription polymerase chain reaction (RT-PCR) showed that the cells expressed many undifferentiated ES cell-marker genes such as Oct3/4, Sox2, Nanog, etc, at levels equivalent to the hES cell line H9 (Figure S1). Moreover, by Western blotting, protein levels of Oct3/4, Sox2, Nanog, etc were similar between the cells and hES cells (Figure S2).

On the other hand, bisulfite genomic sequencing analyses evaluating the methylation status of cytosine guanine dinucleotides $(\mathrm{CpG})$ in the promoter regions of pluripotentassociated genes, such as Oct3/4 and Nanog, revealed that they were highly unmethylated (Figure 4 ). Thus, the cells were more similar to hES cells. We designated the human iPS cells as Chemicals-human induced pluripotent stem (ChiPS) cells.

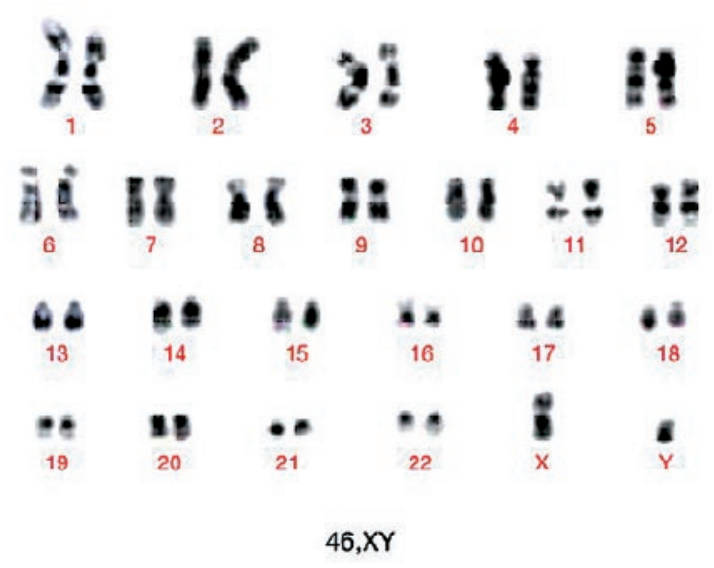

Figure 7. Chromosomal G-band analysis in typical human iPS cells

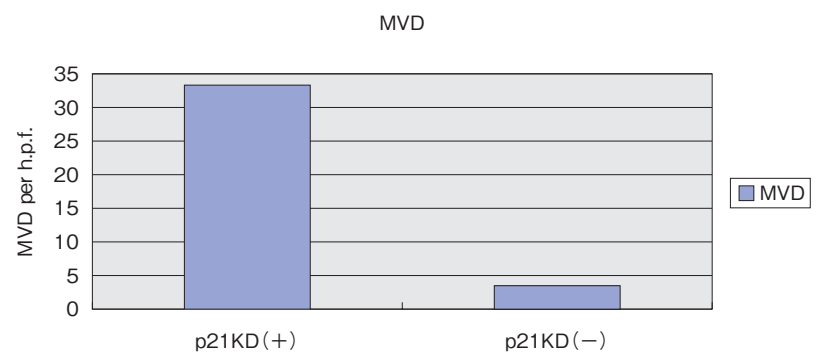

Figure 8. The risk evaluation of malignant transformation for Chips cells as human iPS cell lines

Furthermore, in order to test pluripotency in vivo, we transplanted the ChiPS cell lines as human iPS cell lines subcutaneously into the dorsal flanks of immunodeficient (SCID) mice. Nine weeks after injection, we observed tumor formation. Histological examination showed that the tumor contained neural tissues (ectoderm), striated muscle (mesoderm), and gut-like epithelial tissues (endoderm) (Figure 5), demonstrating that the cells were pluripotent. Moreover, we confirmed the expression of markers for all three germ layers by qRT-PCR analysis (Figure 6). In addition, we could generate human normal hepatocytes from ChiPS cells in vitro, according to method of previous report (Ref. S2).

On the other hand, chromosomal G-band analyses showed that human iPS cells had a normal karyotype of 46 XY (Figure 7). In addition, DNA fingerprinting analysis confirmed that human iPS cells were liver progenitor cells origin (Data not shown). Thus, human iPS cells clones were derived from liver progenitor cells and were not a result of cross-contamination. Therefore we could generate ChiPS cells as human iPS cells from human cells by only small molecules.

The risk evaluation of malignant transformations for the human iPS cells lines

By using MVD within SCID mice that ChiPS cell lines as human iPS cell lines were transplanted, we performed the risk evaluation of malignant transformations for the cell lines (Figure 8). The ChiPS cell lines between the p21 knockdown group and the control group were inoculated intramuscularly into immunodeficient mice (Rag2-/- Il2rg-/-). Then, we compared microvessel density (MVD) within teratomas in mice between the both groups. As a result, the MVD was significantly reduced within teratomas derived from the latter 
compared to the former $(\mathrm{P}<0.01)$. Though cancer risk of patients with down's syndrome was less than healthy people and the MVD in the case of human iPS cells derived from patients with down's syndrome was also less than the MVD in the case of human iPS cells derived from healthy people (4), the MVD in control group of the present study was equal to the case (4) of patients with down's syndrome (Figure 8 and Ref. 4).

Therefore, our observation shows the increased risk for malignant transformations of ChiPS cells by the p21 knockdown (Figure 8), and the induction of p21 is necessary to avoid malignant transformations of ChiPS cells as human iPS cells.

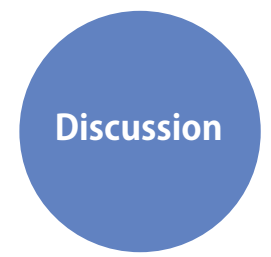

We could generate human iPS cells from human liver progenitor cells by only small molecules. The human iPS cells were similar to hES cells in morphology, proliferation, surface antigens, gene expression, and epigenetic status of pluripotent cell-

\section{- References}

1) $\mathrm{Xu}$ N: MicroRNA-145 regulates Oct 4, Sox2, and Klf4 and represses pluripotency in human embryonic stem cells. Cell 2009; 137: 647-658.

2) Ikushima $H$,Todo $T$, Ino $Y$, et al: Autocrine TGF-beta signaling maintains tumorigenicity of glioma-initiating cells through Sry-related HMG-box factors. Cell Stem Cell 2009; 5: 504-514.

3) Takahashi $\mathrm{K}$, Tanabe $\mathrm{K}$, Ohnuki $\mathrm{M}$, et al: Induction of pluripotent stem cells from adult human fibroblasts by defined factors. Cell 2007; 131: 861-72.

4) Baek KH, Zaslavsky A, Lynch RC, et al: Down's syndo rome suppressions of tumour growth and the role of the calcineurin inhibitor DSCR1. Nature 2009; 459: 1126-1130.

5) Park IH, Zhao R, West JA, Yabuuchi A, Huo H, et al: Reprogramming of human somatic cells to pluripotency with defined factors. Nature 2008; 451: 141-46.

6) Nakagawa M, Koyanagi M, Tanabe K, Takahashi K, Ichisaka $\mathrm{T}$, et al. : Generation of induced pluripotent stem cells without Myc from mouse and human fibroblasts. Nature Biotechnology 2008; 26: 101-6.

7) $\mathrm{Yu} \mathrm{J}, \mathrm{Hu} \mathrm{K}$, Smuga-Otto K, et al: Human induced pluripotent stem cells free of vector and transgenes sequences. Science 2009; 324: 797-801.

8) Moriguchi H, Chung RT, Sato C: An indicator in order to evaluate the risk for cancerous transformations of human induced pluripotent stem cells. Hepatology 2010; 51: 10851086.

9) Mihara M, Nakagawa T, Noguchi S, et al: MRI, Magnetic resonance influenced, organ freezing method under magnetic field. ACSC 2009; 1: 34-37.

S1) Ma S, Lee TK, Zheng BJ, Chan KW, Guan XY: CD133+ HCC cancer stem cells confer chemoresistance by preferential expression of the Akt/PKB survival pathway. Oncogene 2008; 27: 1749-58.

S2) Sullivan GJ, Hay DC, Park IH, et al: Generation of functional human hepatic endoderm from human specific genes. Furthermore, these cells could differentiate into cell types of the three germ layers in vitro and in teratomas. The reprogramming efficiency of generating ChiPS cells as human iPS cells ranged from $0.03-0.05 \%$. Therefore, the efficiency was improved more than previous reports (3) (5) (6) (7).

Moreover, though we previously found the induction of p21 was necessary to avoid malignant transformations of human iPS cells (8), the result was also true of the case of ChiPS cells in the present study.

In conclusion, we try to generate human iPS cells from human normal somatic cells by small molecules used in this study. Perhaps, human iPS cells such as ChiPS cells would be useful in clinical field in the near future. For example, as for children patients with severe cirrhosis, the transplantation of human hepatocytes differentiated from human iPS cells with high quality and safety should be considered. However, further clinical investigations will be needed carefully.

Furthermore, freezing method under magnetic field as our novel technology (9) would be also useful for human iPS cells. The studies are on going in our institutes.

induced pluripotent stem cells. Hepatology 2010; 51: 329335.

\section{-Acknowledgments}

This study was supported by grants from the following organizations.

The authors would like to thank them all:

- Japan Society for the Promotion of Science (Grants-in-Aid for Young Scientists A)

- Japan Interaction in Science and Technology Forum Foundation

- Leave a nest Co., Ltd. (Leave a nest Grant)

- Foundation for Promotion of Cancer Research

- Children's Cancer Association of Japan

- Fukuoka University, School of Medicine Alumni, Eboshikai

- Mitsui Life Social Welfare Foundation

- National Center for Child Health and Development

- Naito Foundation

Furthermore, we are grateful to members of our laboratories for technical supports and Ms. Satoko Iioka for helpful discussions. Moreover, we are grateful to critical reviewers in the world. We have no competing financial interests.

As a policy of ACSC, sales of ACSC are partly contributed to Children's Cancer Association of Japan.

\section{-Author contributions}

H.M.: Conception and design, provision of study material, collection and/or assembly of data, data analysis and interpretation, manuscript writing, final approval of manuscript; C.S.: provision of study material, collection and/or assembly of data, data analysis and interpretation, manuscript assessment, final approval of manuscript; R.T.C: collection and/ or assembly of data, data analysis and interpretation, manuscript assessment, final approval of manuscript; M.M.: manuscript assessment, final approval of manuscript. 